\title{
Spezielle Fördermaßnahmen für die Pädiatrische Onkologie
}

\author{
D. Niethammer \\ Universitäts-Kinderklinik Tübingen
}

In den letzten Jahren ist eine Welle von materieller Hilfe auf die pädiatrische Onkologie zugekommen, deren Ausmaß manchmal kaum noch faßbar ist. Im folgenden soll versucht werden, über die verschiedenen Förderungsmaßnahmen einen kurzen Uberblick zu geben. Es soll deutlich gemacht werden, daß inzwischen Absprachen zwischen den Trägern der verschiedenen Förderungsmaßnahmen bestehen, um auf der einen Seite ein möglichst breites Feld abzudekken und auf der anderen Seite Doppelfinanzierungen zu vermeiden. So werden zum Beispiel im Rahmen der psychosozialen Modellmaßnahme durch den BMA bei der Bewilligung von Stellen bereits vorhandene - von anderer Seite finanzierte Stellen - berücksichtigt und abgezogen. Die Basis für die Förderung und ihre Begründung soll anschließend kurz erörtert und einige Probleme aufgezeigt werden.

\section{Fördermaßnahmen}

\section{a) Privatinitiativen}

Privatinitiativen, ausgehend von betroffenen Einzelpersonen (Dr. Walter Reiners - Kind-Philipp-Stiftung (1972), das Ehepaar John und Erika McDonald - Leukämie-Forschungshilfe (1973)), haben durch ihre großzügiges Engagement frühzeitig zur Unterstützung im Bereich der Forschung und auch Krankenversorgung beigetragen. Entweder werden durch einen eigenen Beirat (Kind-Philipp-Stiftung) oder über die Vorstände von GPO und DAL (Leukämieforschungshilfe) die Mittel vergeben. Hinzugekommen sind in den letzten Jahren noch die Direktleistungen der lokalen Elterninitiativen, die ebenfalls zum Teil früher kaum vorstellbare Ausmaße angenommen haben und mancherorts, sowohl im Bereich der Personalstruktur als auch der räumlichen Gegeben. heiten, maßgebliche Anderungen induziert haben. Aktionen - wie die Tour Peiper - haben die Aufmerksamkeit der Offentlichkeit auf die pädiatrische Onkologie gerichtet.

\section{b) Deutsche Krebshilfe}

Seit Beginn der Deutschen Krebshilfe hat diese die pädiatrische Onkologie in Forschung, Krankenversorgung und Ausbildung (Stipendien) großzügig unterstützt. Während in den ersten Jahren der Vorstand und Beirat eigene Schwerpunkte gesetzt hat, ist zunehmend dazu übergegangen worden, die Förderungen im Bereich der Krankenversorgung in Absprache mit den beiden Gesellschaften vorzunehmen, während die Forschung durch die Dr.-Mildred-Scheel-Stiftung/Deutsche Stiftung für Krebsforschung mit Hilfe und unter der Kontrolle unabhängiger Gutachter unterstützt wird. Auch der Auf- bau der neuen onkologischen Abteilung in Hannover wäre ohne Unterstützung der Deutschen Krebshilfe kaum möglich gewesen.

\section{c) Deutsche Forschungsgemeinschaft}

Die Deutsche Forschungsgemeinschaft hat die Förderung im Bereich der pädiatrischen Onkologie in den Sonderforschungsbereichen 111,112 und 120 wie auch in Einzelverfahren und durch die Gewährung von Stipendien unterstützt und wird dies auch in Zukunft tun. Anträge werden durch unabhängige Gutachter bewertet.

\section{d) Bundesministerium für Arbeit und Sozialordnung (BMA)}

Das Bundesministerium für Arbeit und Sozialordnung war die erste Verwaltungsinstanz, die die Nöte im klinischen Bereich der pädiatrischen Onkologie erkannt hatte. So begann man 1981 eine Förderung von Personalstellen (Ärzte und Schwestern) an einer größeren Zahl von pädiatrischen Zentren. Inzwischen ist die Uberführung dieser Stellen in die Grundausstattung an den meisten Orten gelungen. In Zusammenarbeit mit GPO und DAL wurden dann zwei weitere Problemkreise angegangen, die psychosoziale Betreuung und die Knochenmarktransplantation. Erstmals wurde die Förderung auf eine aufwandsbezogene Basis (Neumeldungen an das Tumorregister in Mainz pro Jahr) gestellt, die in zunehmender Weise auch für andere Förderungen (zum Beispiel Deutsche Krebshilfe, Stern/ZDFSpendenaktion) Geltung haben werden. Durch den BMA wurde auch erreicht, daß die Kinderonkologie als eigener Schwerpunkt in die Bundespflegesatzverordnung mitaufgenommen wurde, was jetzt zur Anerkennung von Sonderpflegesätzen und besseren Personalschlüsseln in vielen Bundesländern geführt hat oder hoffentlich führen wird.

\section{e) Bundesministerium für Forschung und Technologie (BMFT)}

In diesem Zusammenhang darf die Unterstützung der Therapiestudien durch den BMFT nicht vergessen werden. Bei dem hohen Dokumentations- und Auswertungsaufwand in den Studienzentralen wäre die Durchführung der multizentrischen Therapiestudien in den letzten Jahren kaum noch denkbar gewesen. Die Studienförderung, die jetzt zum Teil in andere Hände übergeht, wird mit Sicherheit fortgesetzt und ist eine ganz wesentliche Basis unserer gemeinsamen Arbeit. 
Tab. 1 Förderungsmaßnahmen im Bereich der pädiatrischen Onkologie

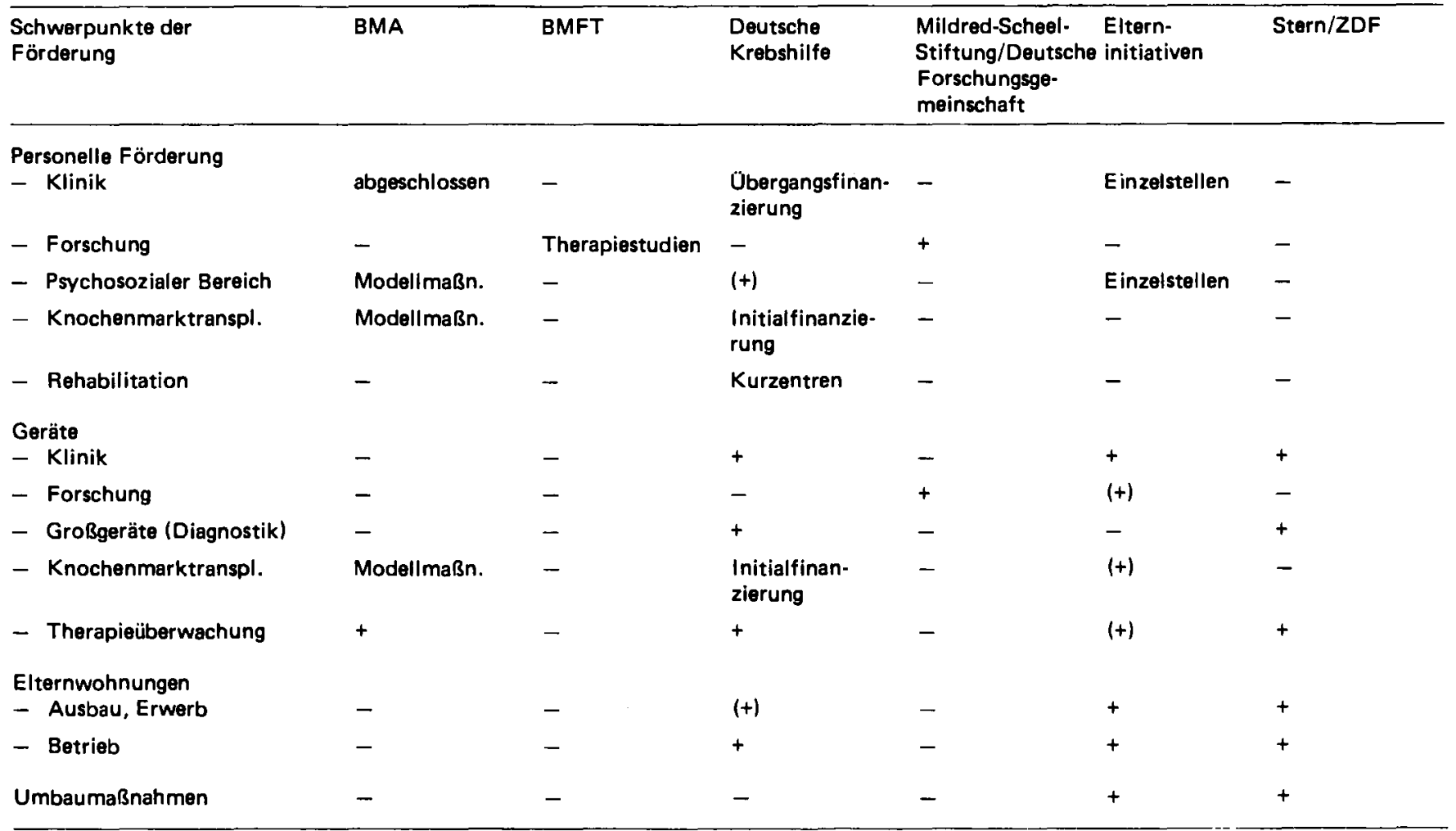

\section{f) Stern/ZDF-Aktion}

Durch eine öffentlichkeitswirksame Aktion dieser beiden Institutionen ist es gelungen, einen Betrag, der die 10 Mill.Grenze deutlich überschreitet, zusammenzutragen und der pädiatrischen Onkologie zur Verfügung zu stellen. Dadurch können patientenorientierte Förderungen dort ausgesprochen werden, wo dies mit keiner der anderen Förderungsmaßnahmen möglich wäre. Dankenswerterweise haben die Träger dieser Aktion beschlossen, die Gelder in Zusammenarbeit mit der Deutschen Krebshilfe, den beiden Gesellschaften (GPO und DAL) und den Elterninitiativen zu ver. geben. Damit wird es möglich, bestimmte Konzepte bei der Förderung zu berücksichtigen und gleichzeitig Doppelförderungen zu verhindern.

\section{g) Stiftung Volkswagenwerk}

Die Erwähnung der Stiftung Volkswagenwerk hat hier leider im Moment nur noch historischen Wert. Jahrelang wurde die pädiatrische Onkologie im Rahmen des Programmes „Das chronisch kranke Kind" unterstützt, wobei das außergewöhnliche Engagement von Frau Brunhilde Mai nicht vergessen werden soll.

In Tabelle 1 sind die verschiedenen Fördermaßnahmen nochmals übersichtlich zusammengefaßt. Es wird das Bemühen deutlich, ein Gesamtkonzept zu entwickeln und Uberschneidungen zu vermeiden.

\section{Probleme der Förderungen}

Eine solche Fülle von Förderungsmaßnahmen in einem kleinen Spezialgebiet - so erfreulich sie auch ist - kann nicht ganz ohne eine gewisse Skepsis betrachtet werden. Es soll im folgenden auf einige Probleme eingegangen werden, über die man in diesem Zusammenhang nachdenken muß.

\section{Grundlagenforschung}

Die großen, bei uns erzielten Fortschritte in der klinischen Onkologie dürfen nicht darüber hinwegtäuschen, daß erhebliche Defizite im Bereich der kliniknahen aber auch der echten Grundlagenforschung bestehen. Dies liegt auf der einen Seite daran, daß in den Kliniken notwendigerweise über viele Jahre Prioritäten zugunsten der Krankenversorgung gesetzt werden mußten. Nur eine relativ kleine Anzahl der Arbeitsgruppen sind eigenständig und genügend groß, so daß der Aufbau von sinnvollen Forschungsprogrammen überhaupt denkbar ist. Aber auch hier sind die freien Valenzen oft viel zu klein - ein Problem, das diese Gruppen natürlich mit praktisch allen anderen klinischen Bereichen an den Universitäten in Deutschland teilen. Strukturen, die - im Gegensatz zum Beispiel in den USA - ein 12-monatiges klinisches Engagement aller Ärzte im Jahr voraussetzen bei praktisch fehlenden Stellen für die Forschung in der Grundausstattung, lassen kaum Freiraum für die Wissenschaft. Eine Geräteförderung zur Unterstützung der Forschung ohne Aufbau einer entsprechenden personellen Infrastruktur erfüllt häufig nicht den Zweck und beinhaltet die Gefahr von Beschaffungsruinen. Gerade aber die Schaffung der personellen Infrastruktur ist durch kurzfristig verfügbare Spendengelder praktisch nicht erreichbar, sondern nur durch eine längerfristige Förderung durch die Deutsche Forschungsgemeinschaft oder die Dr.-Mildred-Scheel-Stiftung denkbar. Voraussetzung für eine qualitativ hochste- 
hende Antragstellung ist aber die Schaffung einer Grundausstattung durch die Träger der Universitäten. Bei den jetzigen Strukturen an den Hochschulen kann es nicht wundern, daß die Zahl der Anträge zu klein oder die Qualität häufig nicht ausreichend ist.

\section{Zentralisierung}

Ein nicht unumstrittenes Problem ist die zunehmende Zentralisierung der Kinder. Für die Patienten und ihre Eltern sind es vor allem die oft langen Anfahrtswege, die notgedrungen zu längeren Trennungen der Familien führen. Dieses Problem ist jedoch in der Regel den anderen Notwendigkeiten unterzuordnen, die durch die aufwendige Diagnostik und die intensiven Therapieverfahren, meist in enger Zusammenarbeit mit anderen Disziplinen, zu einer Zentralisierung in größeren Zentren zwingen, an denen die notwendigen Fachrichtungen vertreten sind. Auch die Weiterentwicklung der Therapie im Rahmen der Studien sowie deren Leitung ist nur in größeren Zentren möglich, die genügend $\mathrm{Er}$ fahrungen durch größere Patientenzahlen aufweisen können.

Neuerdings hat diese Zentralisierung aber noch einen anderen Aspekt bekommen, auf den wir hier näher eingehen müssen, da er eng mit den Förderungsmaßnahmen verknüpft ist. Alle Förderungsinstitutionen sind sich einig, daß eine zeitlich limitierte und punktuelle Förderung von Kliniken, die nur eine kleine Zahl von Patienten behandeln, nicht sinnvoll sein kann. Man kann sich darüber streiten, ob eine Untergrenze von 15 Neuaufnahmen pro Jahr, wie es jetzt festgelegt wurde, der richtige Schnitt ist. Es besteht aber kein Zweifel, daß bei der doch großen Zahl von Krankheitsbildern - es existieren ja alleine Studien für 13 verschiedene Erkrankungen - es schwierig ist, bei der Aufnahme von wesentlich weniger Kinder eine entsprechende Fachkompetenz von Ärzten und Schwestern zu erreichen oder auf Dauer aufrechtzuerhalten.

Die Förderer tragen hier eine große Verantwortung. Die Förderung einer Klinik mit nur wenigen Patienten würde konsequenterweise dem Krankenhausträger den Eindruck vermitteln, Jaß hier ein von den dafür kompetenten Stellen anerkanntes Zentrum besteht, dem gegenüber er ebenfalls Verpflichtungen hat (zum Beispiel durch Anschlußfinanzierung), die er nach Struktur und Aufgabenstellung gar nicht erfüllen kann oder soll. Eine gewisse Zentralisierung hilft auch, Kosten zu sparen. Personal, Geräte sowie auch die Förderung können dadurch gezielter eingesetzt werden und sie kommen so auch mehr Kindern zugute. Auch die Einstellung von Personal im psychosozialen Bereich ist erst ab einer gewissen Patientenzahl sinnvoll. Nur in den Kliniken mit größeren Zahlen von Neuerkrankungen besteht die Chance, daß die jetzt durch den BMA im Rahmen der Modellmaßnahme geförderten Stellen für Mitarbeiter im psychosozialen Bereich auch nach Abschluß des Programms in die Grundausstattung übernommen werden können, damit ihre inzwischen gewonnene Kompetenz nicht wieder verloren geht.

Natürlich muß man auch dringend über Konzepte für die Zusammenarbeit zwischen den Zentren auf der einen Seite und den kleineren Kinderkliniken und auch den niedergelassenen Kinderärzten auf der anderen Seite nachdenken. Nicht immer müssen alle Maßnahmen der Therapie und
Diagnostik im Zentrum gemacht werden. Nach Absprache ist hier eine Verteilung der Arbeit oft sinnvoll und möglich. Gerade die großen Zentren sind hier zu Aktivitäten aufgerufen.

\section{Knochenmarktransplantation}

Noch deutlicher gilt das, was bereits über die Zentralisierung gesagt wurde, für die Knochenmarktransplantation. Die Deutsche Arbeitsgemeinschaft fur Knochenmarktransplantation geht davon aus, daß eine optimale Größe eines Transplantationszentrums zwischen 30 und 60 Transplantationen pro Jahr anzusiedeln ist. Auf längere Sicht werden nur wenige Zentren deutlich darunter oder darüber liegen. Man kann annehmen, daß die Kapazität der bereits vorhandenen und der sich im Aufbau befindlichen Zentren ausreichen wird. Die personelle und apparative Infrastruktur für die allogene Transplantation liegt weit über dem, was bei normalen onkologischen Erkrankungen notwendig ist. Für die autologe Knochenmarktransplantation gilt Vergleichbares, da bis auf die fehlende Graft-versus-Host-Reaktion keine wesentlichen Unterschiede bestehen, ja die Phase der Aplasie im Vergleich zur allogenen Knochenmarktransplantation eher verlängert ist. Hier sind allerdings die notwendigen Kapazitäten nicht ganz so gut abzuscliätzen, da die Entwicklung noch nicht so weit ist.

So hat auch der BMA entschieden, sich an diese Vorstellungen zu halten und bei der Förderung der Knochenmarktransplantationszentren keinen weiteren Neuaufbau von Zentren zu stimulieren. Auch hierbei kann es nicht darum gehen, Ärzten therapeutische Maßnahmen zu verbieten, sondern im Sinne der Kostendämpfung dafür zu sorgen, daß nicht durch kurzzeitige Fördermaßnahmen Gegebenheiten geschaffen werden, die dann notgedrungen zu Anschlußfinanzierungen durch den Krankenhausträger führen müssen. In den USA sind nach neuesten Meldungen bereits 96 Knochenmarktransplantationszentren an der Arbeit und es bestehen Hinweise, daß bereits mancherorts die Notwendigkeit der Auslastung die Indikationsstellung eindeutig beeinflußt. Wenn wir die Forderung an die Krankenhausträger auf Unterstützung unserer Arbeit stellen, dann können sie auch erwarten, daß wir von unserer Seite Konzepte entwickeln, die eine sinnvolle Anwendung der zur Verfügung stehenden knappen Mittel garantiert. Dieser Verantwortung können wir uns nicht entziehen, so unangenehm das auch manchmal ist. Es ist nur sinnvoll, wenn man zunächst in den vorhandenen Zentren die Unzulänglichkeiten beseitigt und die Kapazitäten auf das Machbare erweitert, bevor man neue Zentren gründet, in denen neben den Transplantationseinheiten die gesamte Infrastruktur in den anderen Fächern (Strahlentherapie, Transfusionsmedizin, Virologie, Mikrobiologie) erst aufgebaut werden muß. Hier kann nur eine zentralisierte und bedarfsgerechte Planung eine unsinnige Kostenausweitung verhindern.

Unsere Behandlungserfolge sind erfreulich und ein $\mathrm{Ge}$ schenk, die Mißerfolge bei immerhin noch einem Drittel der Kinder sind Grund zum Ansporn und eine Verpflichtung. Wir haben allen Grund, für die Unterstützung durch verschiedene Menschen und Institutionen dankbar zu sein. Sie legt uns aber auch eine große Verantwortung auf, nämlich die Mittel nicht zu verschwenden und sinnvoll für das 
Wohl unserer Patienten einzusetzen. Daß über die richtigen Wege nicht immer Einigkeit herrscht, liegt in der Natur der Sache, enthebt uns aber nicht der Notwendigkeit, Entscheidungen - nach Möglichkeit unter Erarbeitung von Konzepten - zu treffen, auch wenn diese nicht immer für alle Betroffenen befriedigend sein können.

Prof. Dr. D. Niethammer, Univ.-Kinderklinik, Rümelinstraße 23, D-7400 Tübingen 